\begin{tabular}{|c|c|c|}
\hline & Int.J.Curr.Microbiol.App.Sci (2017) 6(7): $4021-4031$ & \multirow{4}{*}{ 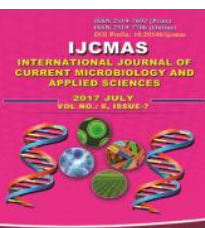 } \\
\hline & \multirow{4}{*}{$\begin{array}{l}\text { International Journal of Current Microbiology and Applied Sciences } \\
\text { ISSN: 2319-7706 Volume } 6 \text { Number } 7 \text { (2017) pp. 4021-4031 } \\
\text { Journal homepage: http://www.ijcmas.com }\end{array}$} & \\
\hline & & \\
\hline EXCELLENT & & \\
\hline PUBLISHERS & & wwww ijemas.com \\
\hline
\end{tabular}

Original Research Article

https://doi.org/10.20546/ijcmas.2017.607.417

\title{
Clonal Evaluation of Eucalyptus Genetic Resources for Pulping Quality
}

\author{
S. Vennila*, K.T. Parthiban and B. Palanikumaran \\ Forest College and Research Institute, Tamil Nadu Agricultural University, \\ Mettupalayam - 641 301, India \\ *Corresponding author
}

\section{A B S T R A C T}

The tree farming is ecologically as well as economically more viable than traditional agriculture. Investment in tree plantations always remained relatively low in India, inspite of the fact that the existing forests cannot continue to meet our wood requirements. However, realizing the existing problem, the expenditure on afforestation has increased enormously from fifth five year plan onwards but still the results on the land are not encouraging and we have not been able to increase area as well as the forest productivity to the desired level. The misery caused to the entire nation due to unprecedented ecodegradation is enormous and warrants immediate remedial measures. To counteract the impending crisis, use of fast growing tree species managed with intensive cultural operations especially in tree farming have opened up new vistas in wood biomass production. Against this backdrop, the current study was planned to screen and identify superior genetic resources of Eucalyptus for higher pulp yield and wood volume. Twenty seven clones in three Eucalyptus species viz., Eucalyptus camaldulensis, Eucalyptus tereticornis and E. urophylla were subjected for pulp quality analysis. The clone EC 48 has been characterized for wood quality towards its amenability for paper and biomass based power generation industries and the results are very encouraging. The clone expressed the Pulp yield of 48\%, Kappa number 19.3 and Lignin content of $23.20 \%$ which expressed superiority over the local seed sources. Similarly, this clone expressed acceptable strength properties viz., Burst index $\left(5.0 \mathrm{~K} \mathrm{~Pa} \mathrm{~m}^{2} \mathrm{~g}^{-1}\right)$, Tear $\left(8.20 \mathrm{~m} \mathrm{Nm}^{2} \mathrm{~g}^{-1}\right)$ and tensile index $\left(80.0 \mathrm{Nm} \mathrm{g}^{-1}\right)$ which are again proved superior. The wood characterization for energy properties indicated that the clone EC MTP 48 had higher Calorific value of $4314 \mathrm{kcal} / \mathrm{kg}$, Fuel value index of 142.58 and Heating value of 30.27 $\mathrm{MJkg}^{-1}$ and extend scope for its amenability towards biomass based energy utility. For biomass based energy utility, the clone can be harvested at the rotation age of 3 years and hence extend greater scope for High Density Short Rotation (HDSR) Energy Plantations. The productivity studies revealed that three clones viz., EC MTP 48, EC MTP 47 and EC MTP 41 recorded superiority in terms of growth characteristics viz., diameter at breast height, height and volume. The phenotypic and genotypic coefficient of variance estimates were low too high in range and volume recorded the highest PCV and GCV. The heritability values were high for all the traits investigated. Considering the pulp quality and productivity conjointly, the clone EC MTP 48 proved superior and this study recommends the suitability of EC MTP 48 for pulpwood plantation programme.

\section{Introduction}

The Indian forests are in a grim situation today with total productivity of only 15 million $\mathrm{m}^{3}$ of industrial timber and 195 million $\mathrm{m}^{3}$ of firewood. The requirement of 
various woods in India by 2020 AD was projected to 437 million tons of fuel wood and charcoal, 37 million $\mathrm{m}^{3}$ of industrial wood, 33 million $\mathrm{m}^{3}$ sawn timbers, 5.7 million $\mathrm{m}^{3}$ pulp and paper wood and 1.3 million tons of wood based panels (Parveen $e t$ al., 2010).

In India, the pulp and paper industry is considered as one of the largest consumers of forest based raw material. The pulpwood is primarily consumed for paper and paperboard production followed by newsprint and rayon making. Application of paper is varied and one cannot think of a life without paper. The paper industry in India comprises of more than 813 mills producing nearly 14.99 million ton of paper, paper board and news print per annum and provides 0.5 million people directly and indirectly employment to 1.5 million people. For the past ten years from 2000 - 2010 paper consumption in India has grown at a compound annual growth rate of 9.93 percent (WWF, 2012). During 20112012, the domestic production of paper and paperboard was 11 million tonnes against the consumption of 11.23 million tonnes $(\mathrm{mt})$, a deficit of $0.23 \mathrm{mt}$. The total production accounts for 1.6 per cent of the world's production. The estimated turnover of the industry was approximately 30,000 to 35,000 crore. The production of paper industry is expected to cross 20 million tons by 2020 and 40 million tons by 2030 with an annual growth rate of 7 - 8 per cent (CRISIL, 2012). Per capita consumption of paper has increased from $5 \mathrm{~kg}$ to $13.2 \mathrm{~kg}$ per annum between 2003 and 2015 against the world average of $40 \mathrm{~kg}$ to $57 \mathrm{~kg}$ (MCI, 2015). An increase in consumption by one kilogram of per capita paper would lead to an increase in demand of one million tons.

There are limited options to meet the evergrowing wood demand either by increasing the total forest covers or increasing productivity of man-made forests substantially. The former happens to be an unachievable target and therefore, the reforestation to be carried out only with genetically improved planting material, which could easily be done either by developing artificial hybrids or clones with substantially higher productivity.

The genus Eucalyptus belongs to the family Myrtaceae and comprises about 700 species (Eldridge et al., 1993). In fact, it is one of the most valuable tree species, which is widely planted across the world due to its wide adaptability, faster growth and short rotation. Eucalypts many wood based industries particularly for pulp, paper, furniture, cellulose and poles as scaffoldings. It finds suitability as a feedstock for the production of cellulosic bio-fuels and production of energy from charcoal, which reduces emission of greenhouse gases (Table 5).

In fact, it sequesters about 10-14 tons of carbon ha per yr from planting to harvesting in fast growing tropical plantations. Furthermore, eucalypts have a positive net carbon balance when computing production of $\mathrm{CO}_{2}$ used for energy from charcoal or as pulp and paper. Eucalypts remove $\mathrm{CO}_{2}$ from the atmosphere at a rate of about 1.8 tone for every tone of dry wood and also generate $\mathrm{O}_{2}$ at a rate of 1.3 tone for every tone of dry wood (Myburg et al., 2006).

Millions of seedlings of Eucalyptus are being planted in India every year but the productivity has not been consummating with the expected yield basically due to poor quality of planting stock. On the other hand, significant improvement in yields has been achieved in many countries through application of breeding and genetic tools coupled with clonal forestry. Hence, there is a need to identify and screen superior short rotation clone for pulpwood, which has the 
potential for high pulp recovery coupled with high productivity.

\section{Materials and Methods}

Eucalyptus tree improvement programme was initiated during 2000 by introducing 135 seed lots from CSIRO Australia and the first generation evaluation was completed during 2005. The first generation evaluation identified 20 potential seed lots (Table 1) and from these base populations 20 plus trees (Clones) have been screened and characterized for pulp and energy quality coupled with their evaluation through clonal means. The clonal evaluation trial was established at Forest College and Research Institute, Mettupalayam using first generation screened 20 clones of three Eucalyptus species viz., Eucalyptus camaldulensis, E. tereticornis and E. urophylla and for comparision 7 local clones and 1 seed sources have been used. From each species, a billet of each $1 \mathrm{~m}$ length and 50-60 $\mathrm{cm}$ girth were collected, debarked and chipped separately and screened. The screened chips were used for pulping experiments. Some chips were converted into dust for proximate chemical analysis. Based on the initial screening study in the laboratory, the wood samples were subjected to analysis of physical and chemical properties. The pulping experiments were also carried out to find out its suitability for papermaking.

The physical characteristics such as bulk density, basic density and moisture content of wood chips are estimated. For the chemical properties analysis, the billets of individual tree species were chipped in pilot chipper; airdried and converted into wood meal. The wood dust passing through 40 mesh but retained over 60 mesh was subjected to analysis for moisture, ash, hot water soluble, one per cent $\mathrm{NaoH}$ soluble, $\mathrm{AB}$ extractive, acid insoluble lignin, pentosans, hollocellulose as per TAPPI methods (TAPPI, 1980). The strength properties such as pulping, identification of kappa number, pulp brightness, paper sheet preparation, paper strength measurement, tensile strength, tearing strength, bursting strength measurement, black liquor analysis were analyzed as per standard method (TAPPI, 1980).

Twenty seven Eucalyptus clones were multiplied at the clonal complex using a coppice shoot cuttings obtained from selected clones. The existing seed source of Eucalyptus hybrid was raised through seeds and used for comparison. The clones and seed source were established in the field in a randomized block design with three replications. In each replication nine ramets/seedlings were used for the experiments. The data on height, diameter and volume index were periodically measured and analysed and the analysis of variance, ANOVA table alone with

Associated $\mathrm{t}$ test were conducted following the methods of Panse and Sukhatme (1978).

\section{Pulp Wood characterization of eucalyptus clones}

\section{Physical properties of wood chips}

The results of the study indicated that the moisture contents of wood sample of all the clones were found to be ranged between 9.76 (EC MTP 47) and 10.90 (EU MTP 8). The bulk density (284 kg m-3) and basic density $(542 \mathrm{~kg} \mathrm{~m}-3)$ were found highest in clone EC MTP 48 and lowest in clone EC MTP 41 (Table 2). It showed that increased density to be strongly linked to favourable strength, stiffness, hardness and working properties of sawn timbers, as well as pulp yield and paper making quality. The wood density of Eucalyptus pulp wood is possibly one of the most influential factors controlling the 
strength and several other physical characteristics of the paper sheet. It is relatively simple and inexpensive property to determine, even in unsophisticated environments. The bulk density exhibited wide variation and the maximum density was recorded by the clone EC MTP 48. This variation among tested clones and seed source may be due to the differences between early and late wood, which could have created variation between and within trees. Similarly significant difference was observed among Eucalyptus species in basic density, which ranged between $446 \mathrm{~kg} \mathrm{~m}^{-3}$ (EC MTP 41) and $542 \mathrm{~kg} \mathrm{~m}^{-3}$ (EC MTP 48). The wood density properties are of major importance for the production of quality pulp and paper. The amount of wood needed to produce one tone of air dried pulp is calculated from the density and pulp yield (Storebraten, 1990). Persson (1975) found that differences in diameter growth have major impact on basic density of wood. Basic density is again highly correlated with late wood content (Bergstedt and Olsen, 2000). Similarly, the variability exhibited in most physical properties studied among different Eucalyptus clones in the current study also attests the results of earlier findings. But it is important to understand the exact relationship between wood density and other fibre characteristics of the test clones that have an effect on pulp and paper quality.

\section{Chemical properties of wood chips}

The proximate chemical analyses give an idea of potentiality of raw material for paper making. The chemical analysis in terms of ash content ranged between 0.32 (EC MTP 48) and 0.71 (EU MTP 1) (Table 3). The chemical investigation carried out in wood pulp of Acacia mangium recorded high ash content (Saepuloh, 1999). However, all the selected clones in the current study exhibited lower ash content, which thus lend a scope for utilization as improved pulp wood. The alcohol-benzene solubilities of wood constitute the waxes, fats and resinous matter. In the current study, the extractives were in the range between 1.1 (EU MTP 1, EU MTP 2 and ET MTP 29) and 1.4 (ET MTP 14, EC MTP 47 and EC MTP 48) and potential differences were recorded among the selected clones. Similar variation in alcohol benzene extractives were observed among various clones of Eucalyptus tereticornis, wherein the extractives ranged between 1.06 and 1.35 (Rao et al., 1999). Among the chemical properties, holocellulose is very important because it is a measure of total carbohydrate content of the wood (Tappi, 2001). The holocellulose constituting cellulose and hemicellulose is the major portion of fibrous raw material. The holocellulose content in the study ranged between 71.6 (S.O) and 75.2 (EC MTP 48) and other Eucalyptus species recorded in between these. The result indicated the superiority of EC MTP 48 over the existing seed source and other clones. The content of pentosans ranged between 13.0 per cent (EC MTP 47) and 18.5 per cent (S.O) and acid soluble lignin was found to be in the range of 23.0 per cent (EC MTP 47) to 25.7 per cent (S.O). The overall chemical analysis revealed that the clone EC MTP 48 is most superior among twenty seven clones, which could be preferred for commercial deployment for pulpwood plantation establishment.

\section{Strength properties of wood chips}

The strength properties of paper are directly associated with cellulose and inter fibre bonding. The clone EC MTP 48 recorded high holocellulose and low lignin content due to increased pulp yield and is good for interfibre bonding and pulp strength. Similar variations among tree species for various strength properties were also recorded in Eucalyptus tereticornis and Eucalyptus grandis (Patil et al., 1997). Within the 
species, the strength properties varied due to age but in the current study variation occurred among clones of same age which indicated the variation might be due to genetic differences. In the current study, satisfactory levels of strength properties was achieved even in five years of growth, which indicated that the clones tested in the current study could be harvested even in five years as against seven years of current practice by the state forest department.

The comparison of pulping results for yield and strength properties of clones of all the species revealed that EC MTP 48 is most superior compared to Control (S.O). Among the three species under test, Eucalyptus camaldulensis recorded higher strength properties compared to other two species. The strength properties viz., tensile index, tear index, burst index and specific coefficient were recorded superior values in EC MTP 48, EC MTP 47 and EC MTP 41. This might be due to superior fibre characteristic of these genotypes. This besides, the chemical requirement to achieve 20 kappa number in this species is only 17 per cent with normal chemical requirement and good bleaching response might also contributed for improved strength properties.

Table.1 Biometric attributes of Eucalyptus seed source evaluation trial at 48 MAP

\begin{tabular}{|c|c|c|c|c|c|c|c|c|c|c|}
\hline \multirow[t]{2}{*}{ Species } & \multirow[t]{2}{*}{ Seed lot } & \multicolumn{3}{|c|}{12 MAP } & \multicolumn{3}{|c|}{24 MAP } & \multicolumn{3}{|c|}{48 MAP } \\
\hline & & $\begin{array}{c}\text { Height } \\
(\mathrm{cm})\end{array}$ & $\begin{array}{c}\text { DBH } \\
(\mathbf{c m})\end{array}$ & $\underset{\left(\mathrm{cm}^{3}\right)}{\text { Volume }}$ & $\begin{array}{l}\text { Height } \\
\text { (cm) }\end{array}$ & $\begin{array}{l}\text { DBH } \\
(\mathbf{c m})\end{array}$ & $\begin{array}{c}\text { Volume } \\
\left(\mathrm{cm}^{3}\right)\end{array}$ & $\begin{array}{l}\text { Height } \\
(\mathrm{cm})\end{array}$ & $\begin{array}{c}\text { DBH } \\
(\mathbf{c m})\end{array}$ & $\begin{array}{c}\text { Volume } \\
\left(\mathrm{cm}^{3}\right)\end{array}$ \\
\hline \multicolumn{11}{|c|}{ Eucalyptus urophylla } \\
\hline EU MTP 1 & $17567 / 23$ & 132.40 & 1.16 & 139.85 & 230.40 & 2.50 & 1130.40 & 504.40 & 4.35 & 7492.44 \\
\hline EU MTP 2 & $17567 / 20$ & 107.80 & 1.07 & 96.88 & 215.60 & 3.45 & 2014.45 & 409.20 & 4.49 & 6475.87 \\
\hline EU MTP 5 & $17567 / 10$ & 142.40 & 0.91 & 92.57 & 261.40 & 2.90 & 1725.72 & 519.80 & 4.59 & 8596.69 \\
\hline EU MTP 8 & $17567 / 4$ & 93.80 & 1.01 & 75.11 & 183.00 & 2.07 & 615.55 & 433.00 & 3.29 & 3679.17 \\
\hline EU MTP 9 & $17567 / 19$ & 102.80 & 0.91 & 66.83 & 205.80 & 2.36 & 899.79 & 382.00 & 3.12 & 2919.06 \\
\hline \multicolumn{11}{|c|}{ Eucalyptus tereticornis } \\
\hline ET MTP 13 & 13399/JD954 & 135.39 & 1.18 & 147.99 & 392.60 & 2.23 & 1532.60 & 619.20 & 2.85 & 3948.12 \\
\hline ET MTP 14 & 16647 & 106.00 & 0.93 & 71.97 & 208.80 & 1.32 & 285.59 & 458.20 & 1.98 & 1410.12 \\
\hline ET MTP 24 & 13398JD930 & 110.00 & 0.66 & 37.61 & 262.80 & 1.78 & 653.64 & 488.40 & 2.44 & 2282.58 \\
\hline ET MTP 29 & 19960/K1320 & 148.00 & 1.03 & 123.26 & 333.60 & 2.25 & 1325.75 & 650.60 & 2.66 & 3613.66 \\
\hline ET MTP 31 & 19960/K1323 & 142.60 & 1.07 & 128.16 & 263.00 & 2.26 & 1054.49 & 558.40 & 2.74 & 3290.91 \\
\hline \multicolumn{11}{|c|}{ Eucalyptus camaldulensis } \\
\hline EC MTP 41 & $18275 / 1387$ & 119.40 & 0.60 & 33.74 & 360.80 & 1.39 & 547.23 & 436.60 & 2.35 & 1892.73 \\
\hline EC MTP 44 & 16551/JD1596 & 167.60 & 0.81 & 86.32 & 239.20 & 1.00 & 187.77 & 301.20 & 1.47 & 510.93 \\
\hline EC MTP 45 & $19965 / 24$ & 131.80 & 0.49 & 24.84 & 245.80 & 0.71 & 97.27 & 341.00 & 1.22 & 398.42 \\
\hline EC MTP 47 & 16551/JD1595 & 135.60 & 1.12 & 133.53 & 281.40 & 1.39 & 426.80 & 325.80 & 1.33 & 452.40 \\
\hline EC MTP 48 & 16551/JD1597 & 163.00 & 0.88 & 99.09 & 424.60 & 2.93 & 2861.44 & 472.20 & 3.15 & 3678.04 \\
\hline EC MTP 50 & 19566/DL1843 & 129.60 & 1.07 & 116.48 & 325.40 & 1.19 & 361.73 & 258.80 & 1.51 & 463.22 \\
\hline EC MTP 53 & $19615 / 5090$ & 188.00 & 1.48 & 323.26 & 333.80 & 1.87 & 916.30 & 384.00 & 2.43 & 1779.97 \\
\hline EC MTP 56 & 19566/DL1840 & 126.80 & 0.60 & 35.83 & 335.00 & 1.26 & 417.50 & 419.40 & 1.99 & 1303.78 \\
\hline EC MTP 57 & 19566/DL1841 & 141.00 & 0.63 & 43.93 & 316.80 & 1.30 & 420.28 & 454.40 & 2.10 & 1573.07 \\
\hline EC MTP 58 & $19615 / 5091$ & 135.20 & 1.20 & 152.83 & 248.00 & 1.27 & 313.99 & 311.80 & 1.70 & 707.37 \\
\hline
\end{tabular}


Int.J.Curr.Microbiol.App.Sci (2017) 6(7): 4021-4031

Table.2 Physical characteristics of different Eucalyptus clones

\begin{tabular}{|c|c|c|c|c|c|c|c|c|c|}
\hline \multirow[b]{2}{*}{$\begin{array}{l}\text { S. } \\
\text { No. }\end{array}$} & \multirow[b]{2}{*}{ Clones } & \multirow[b]{2}{*}{$\begin{array}{l}\text { Moisture } \\
\text { content } \\
(\%)\end{array}$} & \multirow{2}{*}{$\begin{array}{c}\text { Bulk } \\
\text { density } \\
\text { (OD } \\
\text { basis) } \\
\left(\mathrm{kg} \mathrm{m}^{-3}\right)\end{array}$} & \multirow{2}{*}{$\begin{array}{c}\text { Basic } \\
\text { density } \\
\text { (OD } \\
\text { basis) } \\
\left(\mathrm{kg} \mathrm{m}^{-3}\right)\end{array}$} & \multicolumn{5}{|c|}{ Chips classification (\%) } \\
\hline & & & & & $\begin{array}{l}+45 \\
\mathrm{~mm}\end{array}$ & $\begin{array}{c}+8 \mathrm{~mm} \\
\text { (over } \\
\text { thick) }\end{array}$ & $\begin{array}{l}+7 \mathrm{~mm} \\
\text { (accept) }\end{array}$ & $\begin{array}{l}+3 \mathrm{~mm} \\
\text { (pin } \\
\text { chips) }\end{array}$ & $\begin{array}{c}-3 \mathrm{~mm} \\
\text { (dust) }\end{array}$ \\
\hline 1 & EU MTP 1 & 10.49 & 260 & 499 & Nil & 6.1 & 80.1 & 13.2 & 0.6 \\
\hline 2 & EU MTP 2 & 10.76 & 270 & 510 & Nil & 5.9 & 78.5 & 14.8 & 0.8 \\
\hline 3 & EU MTP 5 & 10.02 & 224 & 461 & Nil & 7.1 & 81.2 & 11.3 & 0.4 \\
\hline 4 & EU MTP 8 & 10.90 & 230 & 455 & Nil & 5.7 & 80.4 & 13.5 & 0.4 \\
\hline 5 & EU MTP 9 & 10.10 & 235 & 473 & Nil & 6.4 & 75.3 & 17.6 & 0.7 \\
\hline 6 & ET MTP 13 & 11.20 & 230 & 455 & Nil & 5.5 & 76.8 & 17.2 & 0.5 \\
\hline 7 & ET MTP 14 & 10.61 & 249 & 469 & Nil & 6.2 & 77.8 & 15.4 & 0.6 \\
\hline 8 & ET MTP 24 & 10.34 & 210 & 477 & Nil & 8.3 & 79.6 & 11.8 & 0.3 \\
\hline 9 & ET MTP 29 & 10.47 & 236 & 452 & Nil & 5.2 & 80.9 & 13.5 & 0.4 \\
\hline 10 & ET MTP 31 & 10.91 & 238 & 484 & Nil & 4.6 & 81.5 & 13.4 & 0.5 \\
\hline 11 & EC MTP 41 & 10.22 & 234 & 446 & Nil & 6.5 & 82.6 & 10.1 & 0.8 \\
\hline 12 & EC MTP 44 & 9.93 & 224 & 480 & Nil & 5.1 & 78.6 & 15.7 & 0.6 \\
\hline 13 & EC MTP 45 & 10.12 & 246 & 460 & Nil & 4.9 & 79.2 & 15.2 & 0.7 \\
\hline 14 & EC MTP 47 & 9.76 & 270 & 510 & Nil & 4.4 & 82.8 & 12.4 & 0.4 \\
\hline 15 & EC MTP 48 & 9.98 & 284 & 542 & Nil & 6.5 & 81.8 & 11.3 & 0.4 \\
\hline 16 & EC MTP 50 & 10.97 & 245 & 540 & Nil & 7.2 & 79.9 & 12.1 & 0.8 \\
\hline 17 & EC MTP 53 & 10.49 & 240 & 540 & Nil & 5.8 & 81.5 & 12.3 & 0.4 \\
\hline 18 & EC MTP 56 & 10.61 & 212 & 468 & Nil & 5.1 & 82.7 & 11.6 & 0.6 \\
\hline 19 & EC MTP 57 & 9.73 & 210 & 484 & Nil & 6.7 & 72.6 & 20.2 & 0.5 \\
\hline 20 & EC MTP 58 & 10.64 & 214 & 456 & Nil & 4.6 & 72.6 & 22.3 & 0.5 \\
\hline 21 & FC RI 3 & 10.53 & 238 & 475 & Nil & 6.8 & 78.9 & 13.5 & 0.8 \\
\hline 22 & FC RI 53 & 10.09 & 217 & 470 & Nil & 9.7 & 76.8 & 12.9 & 0.6 \\
\hline 23 & FC RI 56 & 10.88 & 242 & 455 & Nil & 8.9 & 78.4 & 11.9 & 0.8 \\
\hline 24 & FCRI 103 & 10.03 & 210 & 454 & Nil & 5.8 & 82.7 & 11.0 & 0.5 \\
\hline 25 & C 106 & 11.78 & 240 & 484 & Nil & 8.6 & 79.0 & 12.1 & 0.3 \\
\hline 26 & C 413 & 10.82 & 220 & 510 & Nil & 9.4 & 71.1 & 19.0 & 0.5 \\
\hline 27 & C 283 & 9.89 & 206 & 425 & Nil & 6.7 & 75.3 & 17.5 & 0.5 \\
\hline 28 & S.O (Control) & 10.29 & 220 & 455 & Nil & 8.3 & 78.6 & 12.8 & 0.3 \\
\hline
\end{tabular}


Int.J.Curr.Microbiol.App.Sci (2017) 6(7): 4021-4031

Table.3 Proximate chemical composition of different Eucalyptus clones

\begin{tabular}{|c|c|c|c|c|c|c|c|c|}
\hline \multirow[b]{2}{*}{$\begin{array}{l}\text { Sl. } \\
\text { No. }\end{array}$} & \multirow[b]{2}{*}{ Clones } & \multirow[b]{2}{*}{$\begin{array}{l}\text { Ash } \\
\text { content } \\
(\%)\end{array}$} & \multicolumn{2}{|c|}{ Solubility in } & \multirow{2}{*}{$\begin{array}{c}\text { Alcohol } \\
\text { benzene } \\
\text { extractive } \\
(\%)\end{array}$} & \multirow{2}{*}{$\begin{array}{l}\text { Acid } \\
\text { insoluble } \\
\text { lignin } \\
(\%)\end{array}$} & \multirow{2}{*}{$\begin{array}{l}\text { Pentosans } \\
\quad(\text { ash } \\
\text { corrected }) \\
\quad(\%)\end{array}$} & \multirow[b]{2}{*}{$\begin{array}{l}\text { Holocellulose } \\
\qquad \%)\end{array}$} \\
\hline & & & $\begin{array}{c}\text { Hot } \\
\text { water } \\
(\%)\end{array}$ & $\begin{array}{c}1 \% \\
\mathrm{NaOH} \\
(\%)\end{array}$ & & & & \\
\hline 1 & EU MTP 1 & 0.54 & 3.0 & 12.9 & 1.1 & 24.3 & 13.4 & 73.1 \\
\hline 2 & EU MTP 2 & 0.45 & 2.9 & 12.2 & 1.1 & 24.9 & 13.7 & 73.1 \\
\hline 3 & EU MTP 5 & 0.63 & 3.3 & 11.9 & 1.8 & 26.0 & 15.8 & 68.5 \\
\hline 4 & EU MTP 8 & 0.53 & 2.8 & 12.5 & 1.2 & 24.6 & 13.2 & 73.3 \\
\hline 5 & EU MTP 9 & 0.50 & 3.9 & 12.2 & 2.7 & 25.5 & 15.2 & 69.8 \\
\hline 6 & ET MTP 13 & 0.68 & 3.5 & 13.1 & 2.0 & 27.8 & 16.5 & 68.9 \\
\hline 7 & ET MTP 14 & 0.43 & 2.8 & 12.8 & 1.4 & 24.2 & 13.8 & 73.7 \\
\hline 8 & ET MTP 24 & 0.55 & 3.5 & 14.7 & 1.8 & 26.7 & 14.1 & 71.4 \\
\hline 9 & ET MTP 29 & 0.34 & 2.7 & 14.3 & 1.1 & 24.3 & 13.9 & 73.4 \\
\hline 10 & ET MTP 31 & 0.36 & 3.2 & 13.9 & 2.0 & 25.3 & 15.1 & 70.3 \\
\hline 11 & EC MTP 41 & 0.43 & 2.9 & 13.5 & 1.2 & 24.5 & 13.3 & 74.6 \\
\hline 12 & EC MTP 44 & 0.71 & 4.1 & 13.7 & 1.3 & 25.4 & 16.7 & 71.1 \\
\hline 13 & EC MTP 45 & 0.63 & 3.5 & 13.6 & 2.7 & 27.1 & 14.8 & 69.7 \\
\hline 14 & EC MTP 47 & 0.46 & 3.4 & 12.2 & 1.4 & 23.0 & 13.0 & 74.8 \\
\hline 15 & EC MTP 48 & 0.32 & 2.7 & 12.9 & 1.4 & 23.2 & 14.4 & 75.2 \\
\hline 16 & EC MTP 50 & 0.53 & 3.7 & 13.8 & 1.3 & 24.4 & 14.8 & 73.2 \\
\hline 17 & EC MTP 53 & 0.48 & 2.7 & 12.7 & 1.3 & 24.3 & 14.6 & 73.2 \\
\hline 18 & EC MTP 56 & 0.37 & 3.3 & 13.1 & 1.6 & 27.6 & 16.8 & 72.9 \\
\hline 19 & EC MTP 57 & 0.37 & 3.6 & 14.2 & 1.5 & 26.7 & 16.2 & 73.7 \\
\hline 20 & EC MTP 58 & 0.48 & 3.4 & 12.8 & 2.4 & 26.8 & 15.8 & 72.3 \\
\hline 21 & FC RI 3 & 0.36 & 3.2 & 12.5 & 1.6 & 27.7 & 15.2 & 71.1 \\
\hline 22 & FC RI 53 & 0.36 & 3.6 & 13.7 & 2.2 & 27.8 & 17.2 & 70.6 \\
\hline 23 & FC RI 56 & 0.63 & 3.7 & 14.7 & 2.1 & 26.4 & 17.5 & 72.8 \\
\hline 24 & FCRI 103 & 0.34 & 3.8 & 12.2 & 1.9 & 26.9 & 17.2 & 72.8 \\
\hline 25 & C 106 & 0.35 & 3.4 & 13.2 & 1.8 & 27.3 & 17.4 & 72.4 \\
\hline 26 & C 413 & 0.31 & 3.8 & 12.3 & 1.6 & 27.7 & 14.1 & 73.3 \\
\hline 27 & C 283 & 0.46 & 3.9 & 14.5 & 2.2 & 27.2 & 14.2 & 72.9 \\
\hline 28 & S.O (Control) & 0.38 & 3.6 & 14.0 & 1.2 & 25.7 & 18.5 & 71.6 \\
\hline
\end{tabular}

Table.4 Comparison of different Eucalyptus clones with respect yield and strength

\begin{tabular}{|c|c|c|c|c|c|}
\hline \multirow{2}{*}{ Species } & \multirow{2}{*}{$\begin{array}{c}\text { Chemical } \\
\text { charge for 20 }\end{array}$} & \multirow{2}{*}{$\begin{array}{c}\text { Unbleached } \\
\text { pulp yield }\end{array}$} & \multicolumn{3}{|c|}{ Strength properties at 300 ml CSF } \\
\cline { 4 - 5 } & kappa & & $\begin{array}{c}\text { Tear index } \\
(\%)\end{array}$ & $\begin{array}{c}\text { Tensile index } \\
\left(\mathrm{m} \mathrm{Nm}^{2} \mathrm{~g}^{-1}\right)\end{array}$ & $\begin{array}{c}\text { Burst index } \\
\left(\mathrm{Nm} \mathrm{g}^{-1}\right)\end{array}$ \\
\hline EU MTP 1 & 17 & 45.06 & 7.5 & 74.0 & 4.3 \\
\hline EU MTP 2 & 17 & 46.84 & 7.7 & 61.0 & 3.4 \\
\hline EU MTP 8 & 17 & 44.65 & 7.5 & 70.0 & 4.1 \\
\hline ET MTP 14 & 17 & 44.28 & 7.6 & 77.0 & 4.6 \\
\hline ET MTP 29 & 17 & 46.51 & 7.7 & 67.0 & 4.3 \\
\hline EC MTP 41 & 17 & 47.35 & 8.0 & 78.0 & 4.7 \\
\hline EC MTP 47 & 17 & 47.38 & 8.0 & 78.0 & 4.4 \\
\hline EC MTP 48 & 17 & 48.38 & 8.2 & 80.0 & 5.0 \\
\hline EC MTP 50 & 17 & 47.02 & 7.9 & 71.0 & 4.1 \\
\hline EC MTP 53 & 17 & 46.91 & 7.8 & 78.0 & 4.2 \\
\hline S.O (Control) & 17 & 44.00 & 7.8 & 72.0 & 4.5 \\
\hline
\end{tabular}


Table.5 Energy properties of EC MTP 48

\begin{tabular}{|c|l|c|c|}
\hline S.No & \multicolumn{1}{|c|}{ Properties } & Eucalyptus & Prosopis juliflora \\
\hline 1. & Calorific value (kcal/kg) & 4314 & 4860 \\
\hline 2. & Moisture content (\%) & 7.67 & 6.67 \\
\hline 3. & Volatile matter (\%) & 69.25 & 68.17 \\
\hline 4. & Ash content (\%) & 2.48 & 2.83 \\
\hline 5. & Fixed carbon (\%) & 20.60 & 22.50 \\
\hline 6. & Ash Fusion Temperature (\%) & 1267 & 1370 \\
\hline 7. & Ash Deformation Temperature (\%) & 1143 & 1220 \\
\hline 8. & Fuel value index & 142.58 & 207.59 \\
\hline 9. & Heating Value $\left(\mathrm{MJkg}^{-1}\right)$ & 30.27 & 30.56 \\
\hline
\end{tabular}

Table.6 EC MTP 48 growth parameters at different growth periods

\begin{tabular}{|c|c|c|c|c|c|c|c|c|c|c|c|c|}
\hline \multirow[b]{2}{*}{ Clones } & \multicolumn{2}{|c|}{12 MAP } & \multicolumn{2}{|c|}{ 24 MAP } & \multicolumn{2}{|c|}{ 36 MAP } & \multicolumn{2}{|c|}{48 MAP } & \multicolumn{4}{|c|}{ 60 MAP } \\
\hline & $\begin{array}{c}\text { Height } \\
\text { (m) }\end{array}$ & $\begin{array}{c}\text { DBH } \\
(\mathbf{c m})\end{array}$ & $\begin{array}{c}\text { Height } \\
\text { (m) }\end{array}$ & $\begin{array}{c}\text { DBH } \\
(\mathbf{c m})\end{array}$ & $\begin{array}{c}\text { Height } \\
\text { (m) }\end{array}$ & $\begin{array}{c}\text { DBH } \\
(\mathbf{c m})\end{array}$ & $\begin{array}{c}\text { Height } \\
\text { (m) }\end{array}$ & $\begin{array}{l}\text { DBH } \\
(\mathbf{c m})\end{array}$ & $\begin{array}{c}\text { Height } \\
\text { (m) }\end{array}$ & $\begin{array}{l}\text { DBH } \\
(\mathbf{c m})\end{array}$ & $\begin{array}{c}\text { Volume } \\
\left(\mathbf{m}^{3}\right)\end{array}$ & $\begin{array}{l}\text { Volume } \\
\text { (ton/ha) }\end{array}$ \\
\hline EU MTP 1 & 1.87 & $1.18^{* *}$ & 4.25 & 3.16 & 6.43 & 6.50 & 9.14 & 9.37 & 9.44 & 10.38 & 0.0800 & 80.00 \\
\hline EU MTP 2 & 1.96 & $1.27 * *$ & 5.48 & 3.21 & 8.46 & 7.85 & 10.33 & 10.33 & 11.46 & 11.43 & 0.1169 & 116.94 \\
\hline EU MTP 5 & 1.77 & 0.97 & 4.49 & 3.01 & 6.50 & 6.84 & 7.80 & 9.53 & 9.13 & 11.10 & 0.0864 & 86.42 \\
\hline EU MTP 8 & 1.79 & 0.86 & 4.27 & 2.51 & 5.93 & 5.57 & 7.97 & 8.63 & 9.10 & 10.00 & 0.0723 & 72.33 \\
\hline EU MTP 9 & 1.80 & 0.82 & 4.99 & $3.85^{*}$ & 7.37 & $8.92 * *$ & 8.70 & 9.98 & 9.73 & 10.83 & 0.0868 & 86.80 \\
\hline ET MTP 13 & 1.73 & 0.81 & 4.70 & $4.07 * *$ & 7.67 & 7.30 & 9.43 & 9.20 & 10.57 & 10.52 & 0.0893 & 89.31 \\
\hline ET MTP 14 & 1.87 & 0.95 & 5.73 & $5.37 * *$ & $9.20 * *$ & 8.14 & 9.31 & 9.88 & 10.39 & 10.71 & 0.0899 & 89.88 \\
\hline ET MTP 24 & 1.73 & 0.83 & 5.10 & 3.67 & 8.59 & 6.90 & $11.60 * *$ & 9.17 & 12.56 & 10.50 & 0.1059 & 105.95 \\
\hline ET MTP 29 & 1.80 & 0.91 & $6.50 * *$ & $4.03 * *$ & 10.37 & 7.44 & $11.65^{* *}$ & 10.29 & $12.73^{* *}$ & 11.21 & 0.1210 & 121.00 \\
\hline ET MTP 31 & 1.73 & 0.73 & 4.10 & $3.86^{*}$ & 6.30 & 6.23 & 9.01 & 8.71 & 10.29 & 9.70 & 0.0747 & 74.74 \\
\hline EC MTP 41 & $2.03^{*}$ & $1.36 * *$ & 5.23 & 3.51 & 8.13 & 7.11 & 10.60 & 9.77 & 11.68 & 12.01 & 0.1297 & 129.72 \\
\hline ЕС МТР 44 & 1.67 & $1.07 * *$ & 5.33 & 2.38 & $10.37 * *$ & 5.61 & $13.57 * *$ & 8.39 & 14.60 ** & 9.43 & 0.0996 & 99.58 \\
\hline EC MTP 45 & 1.70 & 0.94 & 4.63 & $4.18 * *$ & 7.93 & $9.16^{* *}$ & 9.67 & 11.08 & 10.61 & 12.06 & 0.1164 & 116.43 \\
\hline ЕC МТP 47 & $2.07 * *$ & $1.19^{* * *}$ & 4.97 & $4.55^{* *}$ & $9.77 * *$ & $9.08 * *$ & $12.05^{* *}$ & 10.72 & 13.22 ** & 11.84 & 0.1426 & 142.62 \\
\hline ЕС МТP 48 & $2.43 * *$ & $1.34 * *$ & $7.53 * *$ & $4.12 * *$ & $11.43 * *$ & $8.57 * *$ & $14.97 * *$ & 11.59 & $15.69^{* *}$ & $12.67 *$ & 0.1920 ** & 192.03 \\
\hline EC MTP 50 & 1.93 & 0.94 & $6.43 * *$ & $4.19^{* *}$ & $9.87 * *$ & $8.70 * *$ & $12.84 * *$ & 10.40 & 13.79 ** & 11.25 & 0.1341 & 134.08 \\
\hline EC MTP 53 & 1.80 & 0.96 & $6.70 * *$ & $5.07 * *$ & $9.17 * *$ & $8.53 * *$ & $11.43^{*}$ & 10.31 & 12.14 & 11.57 & 0.1229 & 122.95 \\
\hline EC MTP 56 & 1.77 & 0.77 & 4.83 & 3.61 & $9.27 * *$ & $8.95 * *$ & $12.57 * *$ & 11.13 & $13.50^{* *}$ & 12.18 & $0.1523 *$ & 152.34 \\
\hline EC MTP 57 & 1.73 & 0.81 & 4.50 & $3.84 *$ & 5.97 & $8.63 * *$ & 8.57 & 11.20 & 9.60 & $12.63^{*}$ & 0.1168 & 116.81 \\
\hline EC MTP 58 & 1.70 & 0.90 & 4.05 & 2.92 & 6.02 & $8.80 * *$ & 8.67 & 10.81 & 9.73 & 11.68 & 0.0999 & 99.90 \\
\hline FCRI 3 & 1.73 & 0.93 & $6.31 * *$ & 2.80 & $9.34 * *$ & 8.23 & $12.29 * *$ & 10.59 & $13.33^{* *}$ & 11.44 & 0.1359 & 135.92 \\
\hline FCRI 53 & 1.67 & 0.96 & 5.05 & 3.03 & 8.55 & $8.67 * *$ & 11.30 & 11.17 & $12.40^{*}$ & 12.32 & $0.1461^{*}$ & 146.13 \\
\hline FCRI 56 & 1.87 & 0.91 & 5.42 & 3.13 & $9.48 * *$ & 7.79 & $12.83 * *$ & 10.53 & $13.73 * *$ & 11.64 & 0.1420 & 142.04 \\
\hline FCRI 103 & 1.83 & 0.81 & $6.37 * *$ & 3.04 & $9.40 * *$ & $8.65 * *$ & $11.84 * *$ & 10.53 & $12.83^{* *}$ & 11.67 & 0.1330 & 133.00 \\
\hline C 106 & 1.77 & 0.80 & $6.68 * *$ & 2.94 & $9.31 * *$ & 6.17 & $12.38 * *$ & 9.47 & $13.43 * *$ & 10.38 & 0.1087 & 108.76 \\
\hline C 413 & 1.73 & 0.83 & 5.41 & 3.19 & $9.80 * *$ & $8.68 * *$ & $12.18 * *$ & 11.42 & $13.36^{* *}$ & 12.33 & 0.1540 & 153.99 \\
\hline C 283 & 1.83 & 0.90 & 5.17 & 3.25 & $9.70 * *$ & $8.27 *$ & $13.38 * *$ & 10.32 & $14.64 * *$ & 11.27 & 0.1420 & 142.00 \\
\hline S.O (Control) & 1.57 & 0.71 & 4.01 & 2.58 & 6.64 & 6.05 & 8.69 & 8.81 & 9.72 & 10.08 & 0.0745 & 74.55 \\
\hline Mean & 1.82 & 0.94 & 5.29 & 3.54 & 8.46 & 7.72 & 10.88 & 10.12 & 11.91 & 11.24 & 0.1166 & 116.65 \\
\hline SEd & 0.08 & 0.02 & 0.24 & 0.14 & 0.24 & 0.27 & 0.23 & 0.87 & 0.25 & 0.68 & 0.0138 & \\
\hline $\mathrm{CD}(0.05)$ & 0.17 & 0.04 & 0.48 & 0.29 & 0.48 & 0.54 & 0.47 & 1.75 & 0.50 & 1.36 & 0.0276 & \\
\hline $\mathrm{CD}(0.01)$ & 0.22 & 0.06 & 0.65 & 0.38 & 0.63 & 0.73 & 0.63 & 2.33 & 0.67 & 1.82 & 0.1534 & \\
\hline
\end{tabular}


Among the strength properties, tearing strength depends upon fibre length, width etc. Hence, the maximum tearing strength, burst index and tear index in EC MTP 48, EC MTP 47 and EC MTP 41 must be due to superior fibre characteristics. Strength properties are best obtained with EC MTP 48, EC MTP 47 and EC MTP 41, which might be due to higher freeness and optimized kappa number $(<20)$ recorded by the clones. The wood and wood properties are very important not only for production of paper but also the properties of paper (Storebraten, 1990). The pulp and paper property are highly dependent on fibre morphology and sheet forming processes. Wood with different properties give different pulp and paper qualities (Kibblewhite, 1989). However, in the current study, only dominated trees were selected which expressed wide variability. This indicated the genetic differences among clones of different Eucalyptus species.

Considering all physical, chemical and strength properties, all the trees subjected for analysis were found suitable as a source of pulpwood. However, considering the pulp yield and kappa number coupled with strength properties (Table 4), the superiority of the EC MTP 48, EC MTP 47 and EC MTP 41 as a source of pulpwood was evident and hence the above three clones are recommended for clonal deployment towards establishment of industrial wood pulpwood plantations.

However, the variation in physical, chemical and strength properties observed among the clones of three Eucalyptus species suggest that further improvement could be made via selection, breeding and further clonal deployment of outstanding individuals.

\section{Productivity studies}

Genetically improved and fast growing clonal planting stock has revolutionized productivity and profitability of plantation of many species amenable to vegetative propagation (Lal, 2005). Clonal planting stock is true to type uniform and with all the superior desirable properties of the elite mother tree (Parthiban et al., 2004). It is absolutely necessary to ensure a wide genetic base of clones to safeguard against possible epidemics and to provide continuous superior genetic resources to meet the demands of wood based industries. The existing genetic superiority of elite trees should be evaluated through comparative trial in order to screen site specific clones with superior wood traits. Genetic improvement of the planting stock through clonal evaluation can play a very significant role in improving productivity, yields, quality of produce and profitability. Against this backdrop, clonal evaluation trial was carried out using twenty seven clones and one seed source as control. The clones differed significantly under field conditions during 12, 24, 36, 48 and 60 MAP for various growth parameters such as plant height, diameter at breast height and volume (Table $6)$.

The clonal evaluation trial indicated that one clone viz., EC MTP 48 expressed superiority in all four characters investigated followed by EC MTP 47 and EC MTP 41.Similarly, in Eucalyptus tereticornis four clones viz., ET12, ET9, ET1 and ET6 expressed superiority out of sixteen clones tested (Sasikumar, 2003). Similar results were also reported in the clonal evaluation trials of Eucalyptus grandis (Lambeth et al., 1994). Their study involving sixteen clones and seedling checks indicated that the clones had better yield, straightness and all other growth parameters compared to check lots of seed origin and also significant differences between clones. Vegetative propagation is excellent approach for development of clonal forestry programme (Vivekanandhan et al., 1997) and it helps to exploit non additive 
characteristics (Zobel and Ikemori, 1983). Similar genetic gain by exploiting clonal forestry approaches was also done in the hybrids clones of Eucalyptus grandis and Eucalyptus urophylla. The hybrid vigour was exploited through clonal forestry (Chopra, 2004), which thus lend support to the current investigation. The superiority of few clones in the current study might be due to the genetic that inherited from the selected mother trees. Hence, the three clones viz., EC MTP 48, EC MTP 47 and EC MTP 41, which expressed early superiority could be incorporated in the industrial wood plantation programme.

Considering the wood quality traits coupled with growth attributes the current study recommends clone viz., EC MTP 48 for incorporation in the ongoing plantation programme and also in the future breeding programme.

\section{References}

Bergstedt, A. and P.O. Olesen. 2000. Models for predicting dry matter content of Norway spruce. Scand J. Forest Res., 15: 633-644.

Chopra, R.K. 2004. Tree improvement through clonal forestry-The west coast paper mill experience.In:K.T.Parthiban,M.

Paramathma, K.S. Neelakantan (Eds.), Compendium on clonal forestry NATPFCRI Publication, Coimbatore. p. 250.

Eldridge, K.G., C. Harwood, G. Van Wyk and J. Davidson, 1993. Eucalypts domestication and breeding. New York, Oxford University Press, pp: 288.

Kibblewhite, R.P.1989. New Zealand radiate pine market kraft pulp qualities. PAPRO New Zealand Technical Brochure.

Lal, P. 2005 Integrated development of agroforestry plantations and wood based industries. In: S. K. Chauhan, S.S. Gill,
S.C. Sharma and Rajni Chauhan (Eds.). Agroforestry in 21st century, Agrotech Publishing Academy, Udaipur. pp. 296303.

Lambeth, C.C., Endo, M. and Wright, J. 1994. Genetic analysis of sixteen clonal trials of E. grandis and comparisons with seedling checks. For. Sci. 40(3): 397411.

Ministry of Commerce and Industry, 2015.Annual Report 2015-2016. Department of Industrial Policy \& Promotion, Government of India.

Myburg, Z., D. Grattapaglia, B. Potts, C. Labate, B. Bossinger, M. Byrne, R. Vaillancourt, R. Sederoff and S. Southerton, 2006. Sequencing of the eucalyptus genome: A proposal to DOE-JGI (Available May 2010) http://www.seralliance.com/news/vol3no6/p dfs/proposal.pdf)

Panse, V.G. and P.V. Sukhatme. 1978. Statistical methods for agricultural workers. ICAR Publication, New Delhi.

Parthiban, K.T., M. Paramathma and K.S. Neelakantan. 2004. Compendium on Clonal Forestry. Directorate of Publications, Tamil Nadu Agricultural University, Coimbatore. p. 209

Parveen, A. Kumar, V.K. Sharma and H.S. Ginwal. 2010. Sustained Hybrid Vigor in F1 Hybrids of Eucalyptus torelliana F.v.Muell x E. citriodora Hook. World Applied Sciences Journal 11 (7): 830834.

Patil, J.V., Deshmukh, R.B. Jambhale, N.D., Patil S.C and Kunjir, N.T. 1997. Correlation and path analysis in Eucalyptus. Indian J.For. 22(2): 132135.

Persson, A. 1975. Wood and pulp of Norway spruce and Scots pine at various spacings. Department of Forest Yield Research, Research Notes 37, Royal College of Forestry, Stockholm, Sweden. p. 145. 
Rao, R.V., V. Kothiyal, P. Sreevani, S. Shashikala, S. Naithani and S.V. Singh. 1999. Yield and strength properties of pulp of some clones of Eucalyptus tereticornis. Indian Forester, 125(11): 1145-1151.

Saepuloh, G.P.D. 1999. Chemical component analysis on Mangium wood at its several age groups from Riau. Bulletin Penelitian Hasil-Hutan, 17(3): 140-148.

Sasikumar, K. 2003. Clonal evaluation of Eucalyptus tereticornis Sm. and Acacia hybrids and interspecific hybridization between A. auriculiformis A. Cunn. Ex. Benth. And A. mangium willd. Ph.D. Thesis, Tamil Nadu Agricultural University, Coimbatore.

Storebraten, S. 1990. Sulfatfabrikken virkesforsyningens soppelplass Foredrag i PTF, Masseteknisk gruppe, 9
Oktober, Oslo, Norway. p. 25.

Tappi. 1980. Standard and suggested methods. Technical association of pulp and paper industry, New York. pp. 200265.

Tappi. 2001. Laboratory manual on testing procedures. Published by the Director, Central Pulp and Paper Research Institute, Saharanpur (U.P.). TM 1-A9.

Vivekanandhan, K., Gurumurthi, K. and Jayaraj, R.S.C. 1997. Clonal multiplication of Eucalyptus. Institute of Forest Genetic and Tree Breeding, Coimbatore.

Zobel, B.J. and Ikemori, Y.K. 1983. Vegetative propagation in Eucalyptus. In: Proc. 9th meeting, Canadian Tree Improvement Association.

\section{How to cite this article:}

Vennila, S., K.T. Parthiban and Palanikumaran, B. 2017. Clonal Evaluation of Eucalyptus Genetic Resources for Pulping Quality. Int.J.Curr.Microbiol.App.Sci. 6(7): 4021-4031. doi: https://doi.org/10.20546/ijcmas.2017.607.417 